\title{
Diretrizes metodológicas para investigar estados alterados de consciência e experiências anômalas
}

\author{
Alexander Moreira de Almeida ${ }^{1}$ \\ Francisco Lotufo Neto ${ }^{2}$
}

Recebido: 13/12/2002 Aceito: 3/2/2003

\begin{abstract}
RESUMO
As experiências anômalas (EA) (vivências incomuns ou que se acredita diferentes do habitual e das explicações usualmente aceitas como realidade: alucinações, sinestesia e vivências interpretadas como telepáticas...) e os estados alterados de consciência (EAC) são descritos em todas as civilizações de todas as eras, constituindo-se elementos importantes na história das sociedades. Apesar disso, têm recebido pouca atenção da comunidade científica, ou são abordados de forma pouco rigorosa. As EA e os EAC podem ser estudados sem que se compartilhem as crenças envolvidas, sendo possível investigá-los enquanto experiências subjetivas e, como tais, correlacionados com quaisquer outros dados. Neste artigo, procurou-se apresentar algumas diretrizes metodológicas para um estudo rigoroso do tema, entre elas: evitar uma abordagem preconceituosa e a "patologização" do diferente, a necessidade de uma teoria e de uma revisão exaustiva da literatura existente, utilizar diversos critérios de normalidade e patologia, investigar populações clínicas e não clínicas, desenvolvimento de instrumentos adequados para avaliação, cuidados na escolha dos termos e no estabelecimento de nexos causais, distinguir entre a experiência vivenciada e suas interpretações, considerar o papel da cultura, avaliar a confiabilidade e a validade dos relatos, por fim, o desafio gerado pela necessidade de criatividade e diversidade na escolha dos métodos.
\end{abstract}

Unitermos: Metodologia; Estado alterado de consciência; Espiritualidade; Experiência anômala.

\section{ABSTRACT}

Methodological guidelines to explore altered states of consciousness and anomalous experiences

Anomalous experiences (AE) (uncommon experiences or one that is believed to deviate from the usually accepted explanations of reality: hallucinations, synesthesia, experiences interpreted as telepathic...) and altered states of consciousness (ASC) are described in all societies of all ages. Even so, the scientists have long neglected the studies on this theme. To study AE and ASC is not necessary to share the believes we discuss, they can be investigated as subjective experiences and correlated like any other set of data. This article presents some methodological guidelines to explore these experiences, among them: to avoid prejudice approaches and to "pathologize" the unusual, the value of a theory and a comprehensive review of literature, using various concepts of pathology and normality, the investigation of clinical and non-clinical populations, development of new search instruments, to be careful to choose terms and to decide causal nexus, to distinguish experiences and interpretations, to take into account the role of culture, to evaluate the validity and reliability of reports, and the last, but not least, creativity and diversity in choosing methods.

Keywords: Methodology; Altered states of consciousness; Spirituality; Anomalous experience.

Médico Psiquiatra e Doutorando em Psiquiatria pelo Departamento de Psiquiatria da FMUSP, Fundador e Coordenador do NEPER.

2 Médico Psiquiatra, Livre-Docente do Departamento de Psiquiatria da FMUSP e Fundador do NEPER.

Endereço para correspondência:

Núcleo de Estudos de Problemas Espirituais e Religiosos - NEPER

Instituto de Psiquiatria do Hospital das Clínicas da FMUSP

Rua Dr. Ovídio Pires de Campos, s/no

São Paulo, SP - CEP 050403-010

E-mail: alexma@usp.br 


\section{Introdução}

O termo "experiência anômala" (EA) é empregado para designar uma experiência incomum (ex.: alucinação, sinestesia) ou que, embora seja relatada por muitas pessoas (ex.: vivências interpretadas como telepáticas), acredita-se diferente do habitual e das explicações usualmente aceitas como realidade. Não há necessariamente uma relação com patologia ou anormalidade (Cardeña, Lyinn e Krippner, 2000).

Bastante relacionados com EA estão os "estados alterados de consciência” (EAC), que Charles Tart (1972) definiu como "uma alteração qualitativa no padrão global de funcionamento mental que o indivíduo sente ser radicalmente diferente do seu modo usual de funcionamento". O mesmo autor faz uma analogia entre a mudança de um estado habitual de consciência para um EAC, descrevendo que um mesmo computador pode funcionar com dois programas diferentes. Nessa situação, um mesmo conjunto de dados ou informações pode ser processado de formas muito diferentes e gerar saídas bem diversas.

Embora haja uma sobreposição entre EA e EAC, as primeiras podem ocorrer em estados usuais de consciência (alucinações).

As EA e os EAC são descritos em todas as civilizações de todas as eras, constituindo-se muitas vezes elementos importantes na história das sociedades e, principalmente, de suas religiões. Têm um profundo impacto, tanto na vida daqueles que os vivenciam diretamente quanto naqueles que os acompanham próximos. As dimensões religiosas e espirituais da cultura estão entre os mais importantes fatores que estruturam as crenças, os valores, os comportamentos e os padrões de adoecimento humanos, ou seja, a experiência humana (Lukoff e Turner, 1992). As experiências dissociativas ocorrem muito freqüentemente em contextos religiosos, constituindo-se muitas vezes em EAC. Apesar de serem conceitos específicos, EA, EAC, fenômenos dissociativos e religiosos estão de tal modo interligados que os usaremos de modo mais ou menos intercambiável na presente reflexão.

Freqüentemente, as publicações científicas têm considerado tais vivências como fenômenos raros, vestígios de "culturas primitivas" ou indicadores de psicopatologia (Freud, 1969; Horton, 1974; Mulhern, 1991; Lukoff, 1992; Munro, 1992, Persinger, 1992; Greenberg, 1992). No entanto, várias pesquisas populacionais recentes têm demonstrado que experiências dissociativas (Ross, Joshi e Currie, 1990) e tidas como paranormais (Ross e Joshi, 1992) são muito freqüentes. Neste último estudo, alguma experiência "paranormal" (incluindo telepatia, sonhos precognitivos, deja vu, conhecimento de vidas passadas...) foi relatada por $65 \%$ da população de uma região no Canadá. Em levantamento nos EUA, $17 \%$ da amostra respondeu sim quando interrogado: "Você já se sentiu em contato com alguém que já morreu?" (Gallup e Newport, 1991).

As EA são tão freqüentes na população geral que nenhuma teoria de psicologia normal ou patológica pode ser completa se não levá-las em consideração (Ross e Joshi, 1992). A visão de que essas vivências são raras e patológicas tem sido uma forma de controle social, tornando-se uma profecia que se auto realiza. Como se crê que elas são consideradas óbvios indícios de psicopatologia, apenas os pacientes francamente psicóticos e que perderam a crítica falam abertamente de suas experiências (Hufford, 1992).

Apesar de sua enorme importância, as experiências anômalas, místicas, religiosas e "paranormais" têm sido amplamente negligenciadas pela pesquisa e pela prática psiquiátrica. No fim do século XIX e início do XX o tema foi investigado de modo bastante consistente (James, 1991/1902; Jung 1994/1902; Myers, 1906), mas logo em seguida foi excluído da agenda (Ross e Joshi, 1992). Nas últimas décadas tem havido um reflorescimento das pesquisas nessa área (Grof e Grof, 2001; Lewis-Fernández, 1998).

É importante enfatizar que é possível estudar as EA sem compartilhar das crenças envolvidas, mas é preciso levar a sério suas implicações e não subestimar as razões pelas quais tantas pessoas as professam (Hufford, 1992; King e Dein, 1998). Tais fenômenos podem ser estudados enquanto experiências subjetivas e, como tais, correlacionados com quaisquer outros dados (King e Dein, 1998).

Além do valor cognitivo, de acréscimo ao saber humano, que uma maior compreensão das EA pode trazer, há sérias implicações éticas. Os profissionais de saúde mental não recebem treinamento adequado para lidar com essas questões, mas, como elas ocorrem na prática clínica, estamos atuando fora dos limites de nosso treinamento profissional. Sabe-se que, dependendo da abordagem realizada, uma mesma vivência (experiência de quase morte) pode ser utilizada como estímulo para o crescimento pessoal ou reprimida como um evento bizarro, indicador de instabilidade mental (Turner et al., 1995). Compartilhamos com Cardeña (2000) a esperança de que "no futuro, o clínico possa consultar os manuais diagnósticos como auxílio na definição de até onde uma certa experiência (mística) implica ou não em psicopatologia".

Tendo em vista a importância, bem como a carência de estudos sobre o tema, este trabalho tem por objetivo a discussão de diretrizes metodológicas para o estudo das EA e dos EAC, incluindo religião e espiritualidade. Para uma maior organização didática, optou-se por apresentar tais diretrizes em tópicos. 


\section{Cuidados metodológicos}

\section{Evitar o "preconceito dogmático"}

Apesar de saber da inexistência de uma "observação ateórica" (Popper,1982; Kuhn, 2000), é de suma importância abertura para rever posições e hipóteses. Principalmente numa área pouco estudada de modo rigoroso e legitimamente "científico" como esta, as hipóteses existentes geralmente são aceitas mais com base na autoridade de quem as pronuncia do que nas evidências científicas disponíveis (Hare, 1962). Na área da psiquiatria e da religião, por exemplo, é impressionante ver o quanto certas idéias persistem apesar da abundante evidência em contrário (Lotufo Neto, 1997). No estudo das EA, devese trilhar o estreito caminho entre os dois abismos: o cepticismo pirrônico e a credulidade ingênua e buscar sempre ter abertura para explorar as diversas hipóteses possíveis (Stevenson e Greyson, 1979; Stevenson, 1977).

\section{Buscar uma teoria}

Em qualquer investigação, é muito importante a existência de uma teoria ou pelo menos de um paradigma geral. Ele guia o desenho do estudo, a coleta de dados e sua interpretação (Hertz-Picciotto, 1999; Victora et al., 1997). Na sua ausência, fica-se sem saber quais informações são importantes e devem ser colhidas, escolhendo-se geralmente as mais facilmente acessíveis, que muitas vezes não são as mais relevantes para o entendimento do problema em questão (Kuhn, 2000; Berenbaum et al., 2000). Na área das EA, estamos claramente num período pré-paradigmático no sentido kuhniano, pois "não há qualquer conjunto-padrão de métodos ou de fenômenos" que todos os estudiosos da área "se sintam forçados a empregar e explicar", havendo quase tantas teorias quanto o número de pesquisadores em atividade na área (Kuhn, 2000).

Alguns bons critérios na escolha de uma teoria ou paradigma são: simplicidade, abrangência (capacidade de resolver o maior número possível de problemas), falseabilidade (capacidade de fazer previsões arriscadas passíveis de serem testadas) e potencial heurístico (fertilidade em levar a novas descobertas) (Kuhn, 2000; Popper, 1982; Lakatos, 1970).

Um cuidado especial deve ser tomado para não tentar forçar todos os dados obtidos a se encaixarem numa teoria parcial e/ou concebida prematuramente. Esse é um problema comum, tende-se a enxergar apenas as evidências que confirmam a nossa teoria, e ignoram-se ou se distorcem as evidências em contrário (Popper, 1982; Tart, 1972; Kuhn, 2000). A explicação para um caso específico pode não ser adequada para a maioria dos outros e EA similares podem ter etiologias e mecanismos diferentes. $\mathrm{O}$ mesmo vale para fraudes: o fato de terem demonstrado fraudes em certos relatos de EA não garante que todos os outros sejam igualmente imposturas (Pekala e Cardeña, 2000).

\section{Revisão exaustiva da literatura existente}

Essa é uma das recomendações "óbvias”, mas que precisam ser reiteradas devido ao fato de serem constantemente negligenciadas. Em grande parte das situações, os autores parecem estar sempre "começando do zero", ignoram os trabalhos já realizados ou, no máximo, citam apenas as referências que abonam seu enfoque (Rao e Stevenson, 1979).

Fundamental a revisão sistemática da literatura sobre o tema em estudo (Clarke e Oxman, 2001), procurando abranger não só a psiquiátrica, mas também histórica, sociológica, psicológica e religiosa (Lotufo Neto, 1997). William James (1902/1991) esclarece que, na investigação das experiências religiosas, "não deveremos procurar os documentos humanos mais instrutivos nos campos da erudição especializada", mas, "que os documentos mais interessantes para nós serão os dos homens que mais se distinguiram na vida religiosa e se mostraram mais capazes de fazer uma exposição compreensível de suas idéias e motivos". Esse é um lembrete muito oportuno, pois habitualmente ignora-se ou se desqualifica a literatura produzida pelas próprias comunidades ou indivíduos que vivenciam os fenômenos em estudo.

\section{Evitar "patologizar" o diferente de "anômalo"}

Uma postura de humildade intelectual é essencial, pois a intolerância ao não conhecimento e à dor mental de não saber propicia o aparecimento de respostas e conclusões rápidas (Amaro, 1996). O psiquiatra Osório César (1941) fez uma colocação muito pertinente: "Os psiquiatras são gente desconfiada e que têm o péssimo costume de catalogar, quase sempre com um grão de loucura, as idéias e os fatos que venham ultrapassar as fronteiras do bom senso da época" (grifo no original). Tal foi uma atitude corriqueira na história da psiquiatria, considerar psicopatológica qualquer manifestação fora dos padrões ordinários. É um fato reconhecido o quanto essa postura impediu uma melhor compreensão da mente humana e deu origem a atitudes discriminatórias (Almeida e Almeida, 2000; Moore, 2000).

Atualmente já há um reconhecimento, mesmo pelo DSM-IV, de que a maioria das experiências dissociativas e mesmo alucinatórias que ocorrem dentro de contextos aceitos culturalmente não são evidências de psicopatologia (Cardeña et al., 1994).

Charles Tart (2000, pp. 33-50) enfatiza que o preconceito de que nosso estado ordinário de consciência é algo natural e o único modo de lidar corretamente com a 
realidade é um grande obstáculo à compreensão da natureza da mente e dos estados de consciência. Nossas percepções do mundo e de nós mesmos, bem como nossas reações a eles, são construções semi-arbitrárias. Apesar de terem uma base na realidade física, são dependentes dos recursos da nossa aparelhagem biológica e moldados pelo ambiente cultural onde nos desenvolvemos. Pelo fato de sermos humanos, um grande (mas finito) leque de potenciais nos são disponíveis. Pelo fato de nascermos em uma cultura particular, somente uma pequena porção desses potenciais se realizarão. Cada cultura valoriza e desenvolve um determinado repertório de capacidades e condena, suprime, outros. Cada um de nós é o beneficiário dessa herança cultural e vítima e escravo dessa estreiteza de nossa própria cultura. Assim como quase todas as pessoas, de todas as culturas, em todas as épocas, pensamos que nossa cultura local é a melhor e que as outras são selvagens e atrasadas. Geralmente não percebemos que nosso estado ordinário de consciência é apenas um dentre os muitos possíveis de interpretar e interagir com o ambiente, com suas vantagens e limitações. Cada estado de consciência pode trazer informações adicionais, ajudando-nos a ter uma compreensão mais global de nós mesmos e do mundo em que vivemos. Então, o autor faz um desafio: O método científico será expandido para a investigação dos estados de consciência ou o imenso poder dos EAC continuará a ser deixado nas mãos dos diversos cultos e seitas? (Tart, 1972).

Como bem destacou Glen Gabbard (1982): "É incumbência dos psiquiatras estarem familiarizados com o amplo leque de experiências humanas, saudáveis ou patológicas. Precisamos respeitar e diferenciar as experiências incomuns, mas integradoras, das que são (...) desorganizadoras".

\section{Utilizar diversos critérios de normalidade e patologia}

Não há um critério universalmente aceito para a psicopatologia, e as classificações psiquiátricas têm suas limitações. Muitas não levam em consideração o fato de que as EA podem não se associar necessariamente com transtornos mentais. No DSM-III-R, todas as 12 citações sobre religião no Glossário de Termos Técnicos são utilizadas para ilustrar psicopatologia (Post, 1990). Diversas escalas têm viéses teóricos, avaliando negativamente as vivências espirituais e religiosas. Por exemplo, no Minnesota Multiphasic Personality Inventory (MMPI) respostas positivas a quesitos sobre crença religiosa, oração e experiências da presença de Deus são consideradas evidências de psicopatologia (Batson e Ventis, 1982).

Como cada critério ou escala psiquiátrica tem suas diretrizes teóricas, as EA podem se associar de modos diversos com os vários instrumentos de avaliação existentes.
Recomenda-se a utilização de diversos conceitos de psicopatologia na investigação das EA (Berenbaum et al., 2000). Além dos critérios diagnósticos dos manuais, são de extrema valia as avaliações sobre o desempenho social e o nível de sofrimento do indivíduo envolvido. Uma atenção especial deve ser dada às consequiências de uma dada experiência, pois ela pode ser inicialmente fonte de sofrimento e incapacitação, mas, ao seu término, trazer benefícios como um maior sentimento de bem-estar físico e psíquico. Tal é o caso das denominadas "emergências espirituais" que podem se manifestar como crises (Grof e Grof, 2001).

\section{Avaliar os EAC sob critérios adequados}

Cada EAC tem um padrão de funcionamento que privilegia certos aspectos da realidade. Em todo estado de consciência há uma seleção limitada do amplo leque de potencialidades humanas. Um erro comum é considerar um EAC como "bom" ou "ruim" avaliando-o apenas segundo os critérios mais desenvolvidos em nosso estado habitual de consciência (raciocínio lógico, habilidades matemáticas...). É preciso examinar o funcionamento do EAC sob as condições para as quais ele é normalmente utilizado. Geralmente é inadequado denominar um estado de consciência melhor ou pior que outro, a questão deve ser; melhor ou pior em relação a qual habilidade? (Tart, 2000). O estado de sonolência é péssimo para dirigir um automóvel, mas é o ideal para se conseguir uma boa noite de sono...

\section{Investigar populações clínicas e não clínicas}

A maioria dos estudos sobre espiritualidade e EA foi realizada em populações clínicas. Apesar de terem sua utilidade, tais pesquisas não permitem inferências para a população geral. A população clínica possui uma série de viéses de seleção que a torna não representativa daquela (Sims, 1988). As motivações subjacentes a comportamentos similares podem diferir entre indivíduos clinicamente afetados ou não (Cardeña, 2000).

Um exemplo são as alucinações, que são consideradas um dos sintomas mais clássicos e óbvios de psicopatologia. Entretanto, há mais de um século que levantamentos epidemiológicos têm demonstrado que mais de $10 \%$ da população geral relata a ocorrência de fenômenos alucinatórios ao longo da vida (Sidgwick, 1894. Esses e outros dados apontam para a existência de uma substancial minoria da população que vivencia alucinações. Há grande carência de informações sobre alucinações na população não clínica, sendo muito importante saber como esses diferem dos portadores de transtornos mentais (Bentall, 2000).

Experiências dissociativas e tidas como paranormais são freqüientes na população geral e não se associam a uma maior presença de transtornos mentais (Ross, 1990; Lewis-Fernández, 1998; Hales, 1994; Ross e Joshi, 1992). 


\section{Desenvolver instrumentos adequados para avaliar as crenças e as experiências}

Muitas vezes as vivências são avaliadas de modo inadequado. Por exemplo, sabe-se que a avaliação de religiosidade apenas pela afiliação religiosa de um indivíduo é incompleta e pouco informativa, entretanto, esse foi o método mais empregado na última década (Weaver et al., 1998). A avaliação de religiosidade deve ser sempre multidimensional, o mesmo vale para a maioria das EA. Precisamos desenvolver e refinar escalas para avaliar tais vivências de modo abrangente e confiável.

\section{Cuidado na escolha dos termos que descrevem as experiências}

O repertório oferecido pelo vocabulário ocidental para a descrição de vivências espirituais é extremamente deficitário (Hufford, 1992). A linguagem que usamos para nos comunicar sobre questões da vida diária não é adequada para a descrição das vivências em um EAC, que muitas vezes é tida como inefável. As palavras e as estruturas da nossa língua são ferramentas muito inapropriadas para descrever sua natureza e dimensões, particularmente para aqueles que não as vivenciaram (Grof, 2000).

Faz-se mister um grande cuidado na escolha dos termos. Algumas expressões podem inibir o relato de experiências associadas. Stevenson (1983) argumenta que a palavra "alucinação", por ser muito ligada à instabilidade mental, faz com que as pessoas "normais" evitem descrever suas experiências alucinatórias. $\mathrm{O}$ autor, inclusive, questiona o emprego desse termo para todas as experiências sensórias não compartilhadas em pessoas mentalmente transtornadas ou sãs, e propõe um outro termo, idiofania.

Um outro ilustrativo exemplo vem do levantamento populacional de Gallup e Newport (1991). Nove por cento relataram já ter visto ou estado na presença de um fantasma, mas $17 \%$ disseram já ter estado em contato com alguém que já morreu. Os respondentes podem alegar que fantasma é algo como um assustador lençol branco voador e não um ente querido que já faleceu (Hufford, 1992).

De um modo geral, é sempre melhor evitar termos com implicações causais ou teóricas e preferir as descrições fenomenológicas (Hufford, 1992). Quando se realiza a tradução de um termo de uma língua para outra, esta deve ser baseada nos conceitos envolvidos, e não nas palavras (Alarcón, 1995).

\section{Distinguir a experiência vivenciada de suas interpretações}

Apesar de ambas serem importantes, a descrição fenomenológica e sua posterior interpretação pelo indivíduo, elas podem se relacionar de modo diferente com outras variáveis (Berenbaum, 2000). Quando solicitadas a descrever, não as razões (por que), mas o conteúdo (o que) das suas experiências subjetivas, as pessoas são muito mais precisas (Pekala, 1991).

Muitos dos pacientes que relatam e acreditam terem vivido experiências de quase morte, na realidade não estavam, do ponto de vista médico, perto da morte. Embora suas experiências compartilhem muitas semelhanças com os indivíduos que realmente estavam próximos da morte, há algumas diferenças que têm sido estudadas (Owens et al., 1990).

\section{Considerar o papel da cultura}

$\mathrm{O}$ ato de considerar patológica uma experiência dissociativa particular é grandemente influenciado pela cultura (Lewis-Fernández, 1998). Cultura pode ser definida como um conjunto de significados, normas comportamentais e valores que determinam as visões particulares que grupos humanos têm sobre o mundo e eles próprios (Favazza e Oman, 1978). A cultura pode influenciar a prática clínica de diversas formas: como instrumento explicativo, agente patogênico ou patoplástico, fator diagnóstico e como elemento terapêutico e protetor (Alarcón et al., 1999).

Estudos transculturais podem determinar quais características de uma dada experiência são invariáveis e quais são moldadas pelo ambiente e crenças do indivíduo. Pesquisas nesse sentido têm sido feitas nas experiências de quase morte (Greyson e Stevenson, 1980; Stevenson e Greyson, 1979; Parischa e Stevenson, 1986; Greyson, 2000) e nas alegadas memórias de vidas passadas Stevenson, 1977; Stevenson, 1983.

\section{Postura do pesquisador: neutra, mas empática}

A atitude do investigador pode ter sérias implicações sobre os dados obtidos e sobre o indivíduo que relata suas experiências. Um risco é este "incrementar" seu relato ou então omiti-lo, dependendo se o pesquisador assume uma postura entusiástica ou hostil (Stevenson e Greyson, 1979; Owens et al., 1991). Como as pessoas de um modo geral sabem que os "cientistas" tendem a desqualificar as EA ou considerá-las sinais de instabilidade mental, grande parte dessas vivências são dissimuladas (Hufford, 1992).

Tanto pela sua formação (que tende a fornecer uma visão pejorativa das dimensões religiosas e místicas da vida), como pelo fato de os profissionais de saúde mental serem bem menos religiosos que a população geral, estes têm uma grande dificuldade de empatizar com tais vivências (Lukoff et al., 1992). Uma postura hostil e/ou "psiquiatrizante" diante de muitas experiências e crenças pode trazer graves consequiências para aquele que as vivencia (Lu et al., 1994; APA, 1990). 


\section{Cuidado no estabelecimento de nexos causais}

Uma associação causal é aquela em que a mudança na freqüência ou na qualidade de uma exposição resulta em uma mudança correspondente na frequiência da doença ou desfecho de interesse. Descobrir relações causais, entre fatores de risco e doenças, constitui um objetivo primordial da epidemiologia, pois nos fornece informações imprescindíveis para ações de saúde pública a fim de diminuir os agravos à saúde.

Julgamentos sobre causalidade envolvem dois passos principais:

- se a associação entre exposição e doença encontrada em um dado estudo é válida, ou seja, se não pode ser explicada pelo acaso, viés e variáveis de confusão;

- avaliar se a totalidade das evidências disponíveis aponta para uma relação causal.

Nunca é demais lembrar que não existe teste estatístico que indique uma relação causal, os testes de significância apenas explicitam qual a probabilidade de a associação encontrada em um certo estudo se dever ao acaso. A determinação de causalidade é sempre um julgamento feito pelo pesquisador à luz das evidências disponíveis. Para auxiliar nesse julgamento, Hill (1965) propôs alguns critérios que se tornaram clássicos:

- força da associação: o quanto a exposição aumenta o risco de doença;

- consistência: a mesma associação é encontrada por diferentes pesquisadores através de diferentes métodos;

- especificidade da relação entre exposição e doença;

- temporalidade: a exposição precede o desfecho;

- gradiente biológico: curva dose-resposta;

- plausibilidade biológica;

- coerência com os conhecimentos já existentes sobre a doença;

- evidência oriunda de experimentos;

- analogia: se já existe alguma relação causal semelhante descrita.

Hill enfatiza que esses nove itens são apenas diretrizes, nenhum deles pode ser considerado como evidência inquestionável, nem pode ser julgado condição sine qua non. Uma das principais críticas que se faz aos estudos sobre EA é a falta de plausibilidade biológica ou de coerência com os conhecimentos existentes sobre o mundo. Entretanto, o próprio Hill enfatiza que o que é biologicamente plausível depende do conhecimento biológico do momento, que "a associação que está sendo observada pode ser nova para a ciência e não podemos descartá-la tão facilmente como bizarra demais".

\section{Relatos de casos ou série de casos}

O estudo detalhado das características de um certo número de casos semelhantes e ilustrativos pode ser muito útil para uma exploração inicial e para gerar hipóteses a serem testadas em estudos mais elaborados (Grisso, 1993). William James (1991) enfatiza bastante o valor de estudar os casos extremos, os mais "paradigmáticos", pois eles evidenciam de modo ampliado as principais características do fenômeno investigado. "Devemos atentar para as suas formas completamente evolvidas e perfeitas (...) podemos passar ao largo das suas manifestações mais fracas". A experiência de iniciantes num EAC pode ser muito diferente daquela de um indivíduo já habituado. O principiante está aprendendo a lidar com o EAC a cada nova ocorrência, o que inclui dúvidas, medos e desconhecimento (Tart, 2000, pp. 179-80).

Os casos selecionados devem apresentar os fenômenos de interesse de modo florido e necessitam ser avaliados por meio de diversos métodos para se extrair o máximo de informações possível.

Apesar dessa importância capital no desenvolvimento da ciência, faz-se mister não realizar inferências arbitrárias. Uma prática muito comum é a extrapolação dos achados de um certo caso clínico ou da "experiência clínica" para assumirem o status de fatos científicos comprovados. Urge reconhecer os diversos viéses a que estão sujeitos os achados de nossa experiência clínica. Entre eles, temos o efeito placebo, o retorno à média, a evolução natural da doença e a tendenciosidade do paciente e do examinador na avaliação dos resultados (Guyatt et al., 1986; Sackett, 1985). Além desses, há o fato de que muitos pacientes que não se julgam melhorados com a abordagem recebida simplesmente mudam de profissional, havendo uma tendência a recebermos o retorno daqueles que tiveram melhor evolução. Ou seja, os relatos de casos e a nossa experiência clínica servem para levantar hipóteses a serem testadas, devendo haver muita cautela na avaliação, principalmente, de eficácia de tratamento e prognóstico.

\section{Estudos longitudinais}

Os estudos longitudinais geralmente fornecem bons suportes para inferências causais, pois permitem estabelecer a sequiência temporal de surgimento entre as variáveis associadas. Por exemplo, posso encontrar maior prevalência de transtornos mentais num grupo religioso. Nesse caso, seria muito útil saber o que veio primeiro: a adesão ao grupo religioso ou a psicopatologia? Esse conhecimento nos ajudaria a formular, ou descartar, hipóteses como: o hipotético grupo 
religioso é um desencadeador de transtornos mentais, ou as pessoas acometidas de problemas mentais buscam esse grupo como forma de alívio?

\section{Levar em consideração a complexidade das relações entre EA e psicopatologia}

Berenbaum et al. (2000) dividem em quatro as possíveis relações entre EA e psicopatologia:

- sobreposição entre EA e psicopatologia: a EA em si é considerada a própria enfermidade;

- EA contribui para a psicopatologia: pela própria reação do indivíduo (ex.: ansiedade ou o desenvolvimento de delírios para lidar com experiências perceptuais incomuns) ou do meio, que pode rejeitar a experiência e o indivíduo como bizarros, demoníacos ou loucos;

- psicopatologia contribui para a EA: essa contribuição pode ser de modo direto (uso de substâncias e/ou transtornos de humor gerando alucinações) ou indireto (psicopatologia gerando estresse que pode desencadear EA);

- particularidades do indivíduo que predispõem tanto para EA quanto para psicopatologia: eventos vitais traumáticos, traços de personalidade (abertura para experiências), alterações do lobo temporal, etc.

Poderíamos acrescentar uma quinta forma de associação, a coexistência casual e independente entre EA e psicopatologia.

Como essas relações são complexas, devemos explorar mediadores, moderadores, variáveis de confusão. As associações podem ser categóricas e não contínuas (Putman et al., 1996); ou pode existir um limiar além do qual há uma relação com patologia.

\section{Avaliar confiabilidade e validade dos relatos}

Um dos modos de avaliar a consistência interna dos relatos é tentar obter a mesma informação por meio de perguntas similares ao longo da avaliação (Pekala, 1991).

A validade da medida pode ser determinada pela congruência com outros achados na mesma EA (validade convergente, exemplo: alterações fisiológicas detectadas durante o período relatado como estando em EA), pela capacidade de se diferenciar dos relatos de outros tipos de EA (validade discriminante) e pela consistência com outros relatos do mesmo tipo de EA (Pekala e Cardeña, 2000).

\section{Criatividade e diversidade na escolha dos métodos}

A associação de estratégias investigativas, como métodos quantitativos e qualitativos; auto-relatos e entrevistas; psicológico, psicobiológico e socioecológico, entre outros, serão de extrema valia para obtermos uma avaliação mais acurada dos fenômenos em estudo.

As EA, inúmeras vezes, desafiarão nossos conhecimentos e engenhosidade científica. Freqüentemente, elas poderão não ser adequadamente investigadas pelos desenhos tradicionais de estudos (Berenbaum, 2000). Sem perder o rigor científico e o senso crítico, será necessário desenvolver novas abordagens. A tarefa de criar novos paradigmas metodológicos é a empreitada daqueles que se aventuram por um caminho ainda pouco trilhado.

\section{Referências bibliográficas}

Alarcón, R.D. - Culture and Psychiatric Diagnosis. Psychiatric Clinics of North America 18(3): 449-65, 1995.

Alarcón, R.D; Westermeyer, J.; Foulks, E.F.; Ruiz, P. - Clinical Relevance of Contemporary Cultural Psychiatry. J Nerv Ment Dis 187: 465-71, 1999.

Almeida, A.M.; Almeida, A.A.S - "História da Loucura Espírita" no Brasil". XVIII Congresso Brasileiro de Psiquiatria e Regional Meeting World Psychiatric Association. Associação Brasileira de Psiquiatria e World Psychiatric Association, 25-28 out. 2000.

Amaro, J.W.F. - Psicoterapia e Religião. Lemos Editorial, São Paulo, 1996.

American Psychiatric Association - Diagnostic Statistical Manual, 4th ed, Washington DC, American Psychiatric Press, 1994.

APA (American Psychiatric Association) - Guidelines Regarding Possible Conflict Between Psychiatrists' Religious Commitments and Psychiatry Practice. Am J Psychiatry 147: 542, 1990.

Batson, C.D.; Ventis, W.L. - The Religious Experience. Oxford University Press, New York; 1982.

Bentall, R.P. - Hallucinatory Experiences. In: Cardeña, E.; Lyinn, S. J. \& KRIPPNER, S. Varieties of Anomalous Experience: Examining the Scientific Evidence. Washington DC, American Psychological Association, 2000.

Berenbaum, H.; Kerns, J.; Raghavan, C. - Anomalous Experiences, Peculiarity and Psychopathology. In: CARdeña, E.; Lyinn, S. J. \& KRIPPNER, S. Varieties of Anomalous Experience: Examining the Scientific Evidence, Washington DC, American Psychological Association, 2000.

Cardeña, E.; Lewis-Fernández, R.; Bear, D.; Pakianathan, I; Spiegel, D. - Dissociative Disorders. In: DSM-IV Sourcebook, DC, Washington American Psychiatric Press, 1994.

Cardeña, E.; Lyinn, S.J.; Krippner, S. - Varieties of Anomalous Experience: Examining the Scientific Evidence, DC, Washington American Psychological Association, 2000.

CÉSAr, O. - Fenomenologia Supranormal. Revista Paulista de Medicina 19(5): 49-73, 1941.

Clarke, M.; Oxman, A.D. (eds.) - Cochrane Reviewers Handbook 4.1.4 [updated October 2001]. In: The Cochrane Library, Issue 4, 2001. Oxford: Update Software. Updated quarterly.

Favazza, A.; Oman, M. - Overview: Foundations of Cultural Psychiatry. Am J Psychiatry 135: 293-303, 1978.

FREUd, S. - Mal-Estar na Civilização. In: Edição Standard das Obras Psicológicas Completas de Sigmund Freud (1969). Imago Editora. Edição Eletrônica em CD-ROM.

Gabbard, G.O.; Twemlow, S.W.; Jones, F.C. - Differential Diagnosis of Altered Mind/Body Perception. Psychiatry 45: 361-9, 1982.

Gallup GH JR.; Newport, F. - Belief in Paranormal Phenomena Among Adult Americans. Skeptical Inquirer 15: 137-46, 1991. 
Greenberg, D.; Wiztum, E.; Buchbinder, J.T. - Mysticism and Psychosis: The Fate of Bem Zoma. Br J Med Psychology 65: 223-35, 1992.

Greyson, B.; Stevenson, I. - The Phenomenology of Near-Death Experiences. Am J Psychiatry 137: 1193-6, 1980.

Grisso, J.A. - Making Comparisons. Lancet 342: 157-60, 1993.

Grof, S. - Psychology of the Future - Lessons form Modern Consciousness Research - New York. State University of New York Press, pp. 276-7, 2000.

Grof, S.; Grof, C. - Emergência Espiritual: Crise e Transformação Espiritual. Editora Cultrix, São Paulo, 2001.

Guyatt, G; Sackett, D. et al. - Determining Optimal Therapy: Randomized trials in individual patients. N Engl J Med 314: 889-92, 1986.

Hales, R.H. - Psychiatric System Interface Disorders. In: DSM-IV Sourcebook. Washington DC. American Psychiatric Press, 1994.

HARE, E.H. - Masturbatory Insanity: The History of an Idea. The Journal of Mental Science 108: 2-25, 1962 (apud Lotufo Neto, 1997).

Hertz-Picciotto, I. - What You Should Have Learned about Epidemiologic Data Analysis. Epidemiology 10: 778-83, 1999.

Hill, A.B. - The Environment and Disease: Association or Causation? Proc R Soc Med 58: 295-300, 1965.

Horton, P.C. - The Mystical Experience: Substance of an Illusion. Am Psychoanal Assoc J 22: 364-380, 1974.

Hufford, D.J. - Commentary. Paranormal Experiences in the General Population. J Nerv Ment Dis 180: 362-8, 1992.

James, W. - As Variedades da Experiência Religiosa. Um Estudo Sobre a Natureza Humana. Editora Cultrix, São Paulo, 1991.

Jung, C.G. - Sobre a Psicologia e Patologia dos Fenômenos Chamados Ocultos. In: Jung, C.G. Estudos Psiquiátricos. Vozes, Petrópolis, pp. 15-96, 1994.

KING, M.B.; DeIn, S. - The Spiritual Variable in Psychiatric Research. Psychological Medicine 28: 1259-62, 1998.

KIRmayer, L.J. - The Fate of Culture in DSM-IV. Transcultural Psychiatry 35: 339-42, 1998.

Kunn, T.S. - A Estrutura das Revoluções Científicas. Editora Perspectiva, São Paulo, 2000.

Lakatos, I.; Musgrave, A. - Criticism and the Growth of Knowledge. Cambridge University Press, Cambridge, 1970.

LewIS-FernÁnDEZ, R. - A Cultural Critique of the DSM-IV Dissociative Disorders Section. Transcultural Psychiatry 35: 387-400, 1998.

Lotufo Neto, F. - Psiquiatria e Religião: a Prevalência de Transtornos Mentais entre Ministros Religiosos. Tese (livre-docência), Faculdade de Medicina da Universidade de São Paulo. São Paulo, 1997.

Lu, F.G.; LukofF, D.; Turner, R. - Religious or Spiritual Problems. In: DSM-IV Sourcebook. American Psychiatric Press, Washington DC, 1994.

Lukoff, D.; Lu, F.; Tuner, R. - Toward a More Culturally Sensitive DSM-IV: Psychoreligious and Psychospiritual Problems. J Nerv Ment Dis 180: 673-82, 1992.

Lukoff, D.; Lu, F.G.; Turner, R. - Cultural Considerations in the Assessment and Treatment of Religious Problems. Psych Clin N Am 18: 467-85, 1995.

Moore, L.J. - Psychiatric Contributions to Understanding Racism. Transcultural Psychiatry 37: 147-82, 2000.

Mulhern, S. - Embodied Alternative Identities: bearing witness to a world that may have been. Psych Clin N Am 14: 769-86, 1991.

Munro, C.; Persinger, M.A. - Relative Right Temporal-lobe Theta Activity Correlates with Vingiano's Hemispheric Quotient and the "Sensed-Presence". Perceptual and Motor Skills 75: 899903, 1992.

Myers, F.W.H. - Human Personality and Its Survival, 2 vols. Owens, 1906.
Owens, J.E.; Cook, E.W.; Stevenson, I. - Features of "near-death experience" in Relation to Whether or not Patients Were Near Death. Lancet 336: 1175-7, 1990.

Owens, J.E.; Cook, E.W.; Stevenson, I. - Near-Death Experience. Lancet 337: 1167-8, 1991.

Parischa, S.; Stevenson, I. - Near-Death Experiences in India. J Nerv Ment Dis 174: 165-70, 1986.

PeKalA, R.J. - The Phenomenology of Consciousness Inventory (PCI). West Chester, PA: Mid Atlantic Educational Institute, 1991.

PeKala, R.J.; Cardeña, E. - Methodological Issues in the Study of Altered States of Consciousness and Anomalous Experiences. In: CARDEÑA, E.; LyInN, S.J.; KRIPPNER, S. Varieties of Anomalous Experience: Examining the Scientific Evidence. Washington DC, American Psychological Association, 2000.

Persinger, M.A. - Right Hemisphericity, Low Self-esteem, and Unusual Experinces: a Response to Vingiano. Perceptual and Motor Skills 75: 568-70, 1992.

Popper, K.R. - Conjecturas e Refutações. Brasília, Universidade de Brasília, 1982.

Post, S.G. - DSM-III-R and Religion (letter). Am J Psychiatry 147: 813, 1990.

Putnam, F.W.; Carlson, E.B.; Ross, C.A. et al. - Patterns of Dissociation in Clinical and Nonclinical Samples. J Nerv Ment Dis 184: 673-79, 1996.

Rao, D.; Stevenson, I. - Telepathy in Shared Dreams? Am J Psychiatry 136: 1345-6, 1979.

Ross, C.A.; Joshi, S. - Paranormal Experiences in the General Population. J Nerv Ment Dis 180: 357-61, 1992.

Ross, C.A.; Joshi, S.; Currie, R. - Dissociative Experiences in the General Population. Am J Psychiatry 147: 1547-52, 1990.

Sackett, D.; Haynes, R.B.; Tugwell, P. - Clinical Epidemiology: a Basic Science for Clinical Medicine. Little, Brown, Boston, 1985.

Schouten, S.A.; Stevenson, I. - Does the Socio-Psychological Hypothesis Explain Cases of reincarnation Type? J Nerv Ment Dis 186: 504-6, 1998.

Sidgwick, H. - Report on the Census of Hallucinations. Proceedings of the Society for Psychical Research 10: 25-252, 1894. (Apud Berrios, G. E. - The History of Mental Symptoms. Cambridge University Press, Cambridge, 1996)

Sims, A. - Symptoms in the Mind. Ballière Tindall, London, 1988.

Stevenson, I.; Greyson, B. - Near-Death Experience: Relevance to the Question of Survival after Death. JAMA 242: 265-7, 1979.

Stevenson, I. - American Children who Claim to Remember Previous Lives. J Nerv Ment Dis 171: 742-8, 1983.

Stevenson, I. - Do We Need a New Word to Supplement "Hallucination"? Am J Psychiatry 140: 1609-11, 1983.

Stevenson, I. - The Explanatory Value of The Idea of Reincarnation. J Nerv Ment Dis 164: 305-26, 1977.

TART, C.T. - States of Consciousness and State-Specific Sciences. Science 176: 1203-10, 1972.

TART, C.T. - States of Consciousness. Lincoln, Authors Guild Backprint.com Edition, 2000 (1983).

Turner, R.P.; LukofF, D.; Barnhouse, R.T.; Lu, F.G. - Religious or spiritual problem. A culturally sensitive diagnostic category in the OSM-IV. J Nerv Ment Dis 183(7): 435-44, 1995.

Victora, C.G.; Huttly, S.R.; Fuchs, S.C.; Olinto, M.T.A. - The Role of Conceptual Frameworks in Epidemiological Analysis: a Hierarchical Approach. International Journal of Epidemiology 26: 224-7, 1997.

Weaver, A.J.; SAmford, J.A.; Larson, D.B. et al. - A Systematic Review of Research on Religion in Four Major Psychiatric Journals: 1991-1995. J Nerv Ment Dis 186: 187-9, 1998. 